\title{
A method for generation of finite amplitude pulse of sinusoidal sound in free space
}

\author{
Toshiaki Nakamura and Akira Nakamura \\ Institute of Scientific and Industrial Research, Osaka University, \\ 8-1, Mihogaoka, Ibaraki, Osaka, 567 Japan
}

(Received 11 November 1982)

\begin{abstract}
Nonlinear response of acoustic materials to a large amplitude sound depends upon the incident waveform itself. For this reason, we intend to control the radiated waveform of finite amplitude in free space. In order to control the radiated waveform, the waveform control in a pipe was performed by the use of the loudspeaker driven by the computed waveform, and generated wave was radiated from an exponential horn connected to the end of the pipe. However, the observed waveform in free space was different slightly from expected one. As the result of the simulation for the sound transmission through the exponential horn, it became clear that the reflection of the mouth of the horn affected the radiated waveform in free space. Therefore, the waveform control in the pipe was performed by taking account of the effect of the horn and then the result obtained agreed approximately with the waveform expected.
\end{abstract}

PACS number: 43. 20. Px, 43. 25. Cb, 43. 88. Ew

\section{INTRODUCTION}

When the response of acoustic materials to the finite amplitude wave is studied, the waveform impinged on the material is an important factor because nonlinear behaviour of the material depends upon the incident waveform itself. Therefore, it is desirable to use the sound source with capability which can generate the finite amplitude wave with an appropriate waveform for the purpose of the investigation. In our previous report, ${ }^{1)}$ we generated a single pulse of sinusoidal form of one period with finite amplitude in a circular pipe by the use of the loudspeaker driven by the computed waveform and obtained satisfactory results.

With these points as background, we intend to radiate the sinusoidal pulse of finite amplitude in free space in the present paper. Several reports about the waveform control in pipe ${ }^{2,3)}$ and free space $^{4-6)}$ have been made. However, these methods of the waveform control are based on the theory of linear system and cannot be applied to the nonlinear case which is the generation of the finite amplitude wave. In this paper, we generated single pulse in a pipe first by means of the waveform control of finite amplitude, as described in our previous paper, ${ }^{1)}$ and subsequently radiated the controlled waveform into free space from the exponential horn connected to the end of the pipe, after the propagation of appropriate distance into the pipe.

\section{EXPERIMENT}

\subsection{Experimental Apparatus}

As shown in Fig. 1, experimental apparatus is constructed by a driver unit (JBL 2441), a straight pipe with the length of $90 \mathrm{~cm}$, an exponential horn (JBL 2311), and two microphones (B\&K 4138 as Mic. 1 and B\&K 4135 as Mic. 2). The propagation distance of $90 \mathrm{~cm}$ in the pipe is necessary as a minimum length in order to control the stable waveform in the pipe. JBL 2311 horn has the diameter of the mouth of $10 \mathrm{~cm}$ and the flare constant of 0.105 , and is mounted in a square baffle with the length of $114 \mathrm{~cm}$ at the side. 


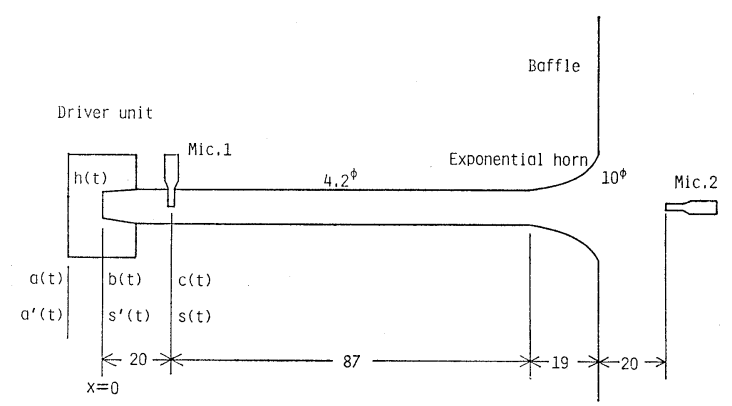

Fig. 1 Schematic diagram of experimental apparatus.

The waveform radiated from a circular piston in infinite baffle is the differential waveform of the piston velocity on axis in far field as it is well known. If we suppose the piston vibration at the boundary on the mouth of the horn without reflection, the differential waveform of sound pressure appeared in the pipe is given on axis in the far field. This is an ideal case without the reflection at the boundary. Accordingly, when a sound pulse with one period of sinusoidal waveform is needed to generate in free space, the sound wave with the integrated waveform of this wave must be generated in the pipe. This means a squared sine wave appeared in the pipe as an integrated wave. From a point of view, the waveform control is performed in pipe so that the squared sine wave is obtained at the position of the throat of the horn. Then, it may be expected that one may observe a sound pulse with sinusoidal form by the microphone placed on axis of the horn in free space. The discussion described above is just valid for case under the assumption of an infinitesimal sound wave. In this paper, however, it is desired that a regular shape of finite amplitude sound is realized in the free space. In this case, we have to take account of the waveform distortion caused by the nonlinear effect when the finite amplitude wave propagates through all the configuration of this experiment, that is, circular pipe, horn and in free space. For this reason, the correction of the waveform distortion is done in ad hoc way as described later. The reflection effect will be also described later.

\subsection{Method of Waveform Control}

As shown in Fig. 1, the point of $x=0$ is located at the position of the sound source realized by the driver unit. When the input signal to the loudspeaker is $a(t)$, the output waveform at $x=0$ is $b(t)$, and the waveform at the position of Mic. 1 is $c(t)$, the relationship among them are written as

$$
\begin{aligned}
& b(t)=\mathrm{NV}[a(t)], \\
& c(t)=\mathrm{NP}[b(t)],
\end{aligned}
$$

where NV and NP represent nonlinear operator for the nonlinear vibration of the loudspeaker and the nonlinear propagation between the sound source and the microphone position respectively. In these two operations, NP is able to be calculated by the method of the simulation described in the previous paper. ${ }^{7)}$ When the desired waveform to be generated at the position of Mic. 1 is $s(t)$, the waveform $s^{\prime}(t)$ written as

$$
s^{\prime}(t)=\mathrm{NP}^{-1}[s(t)]
$$

is necessary to appear at $x=0$ in order to realize $s(t)$ at the microphone position, where $\mathrm{NP}^{-1}$ represents an inverse operation of NP. On the other hand, NV is unknown operator, because the nonlinear vibration of the loudspeaker cannot be described exactly. Therefore, we first suppose that NV is a linear operation and will be introduced its nonlinearity experimentally later. Then, as the response of the driver unit is approximated by the linear operation, Eq. (1) is reduced to the linear relationship which is written by the impulse response $h(t)$ of the driver unit and the convolution integral $(*)$ as follows,

$$
b(t)=h(t) * a(t) .
$$

The transfer function $H(\omega)$ of the driver unit is calculated from Eqs. (2) and (4), written as

$$
H(\omega)=\frac{\mathscr{F}\left\{\mathrm{NP}^{-1}[c(t)]\right\}}{\mathscr{F}\{a(t)\}},
$$

where $\mathscr{F}$ represents Fourier transformation. The waveform $s^{\prime}(t)$ at $x=0$ can be realized by applying the inverse characteristic of $H(\omega)$ to $S^{\prime}(\omega)=\mathscr{F}\left\{s^{\prime}(t)\right\}$, that is

$$
a^{\prime}(t)=\mathscr{L}^{-1}\left\{\frac{S^{\prime}(\omega)}{H(\omega)}\right\},
$$

Therefore $a^{\prime}(t)$ is real input signal to the driver unit in order to realize the waveform $s^{\prime}(t)$ at $x=0$ and $s(t)$ at Mic. 1 position. The transfer function $H(\omega)$ is obtained experimentally for the finite amplitude signal of $a(t)$ in order to avoid the nonlinear effect of the loudspeaker. 


\section{T. NAKAMURA and A. NAKAMURA: GENERATION OF SINUSOIDAL PULSE IN FREE SPACE}

\subsection{Results}

In order to generate a squared form of sinusoidal wave with the width of $0.5 \mathrm{~ms}$ and the peak pressure of 20 mbar shown by the solid line in Fig. 2 at the point of the throat of the horn, the corresponded waveform shown by the broken line in Fig. 2 should be given at the Mic. 1 position. This broken lined waveform is obtained by the same procedure as Eq. (3) which propagates inversely through the pipe from the horn throat to the Mic. 1 position. And then this waveform is modified by the $H(\omega)$ obtained by Eq. (5), as shown in Eq. (6). When the waveform shown in Fig. 3 calculated by Eq. (6) is input to the driver unit, the waveform shown in Fig. 4 was observed by Mic. 1 and this waveform was very similar to the broken lined waveform of Fig. 2. On the axis at the distance of $20 \mathrm{~cm}$ from the mouth of the horn, the waveform shown in Fig. 5 was observed by Mic. 2. This waveform should be simulated by taking account of the horn characteristic as described in the next chapter.

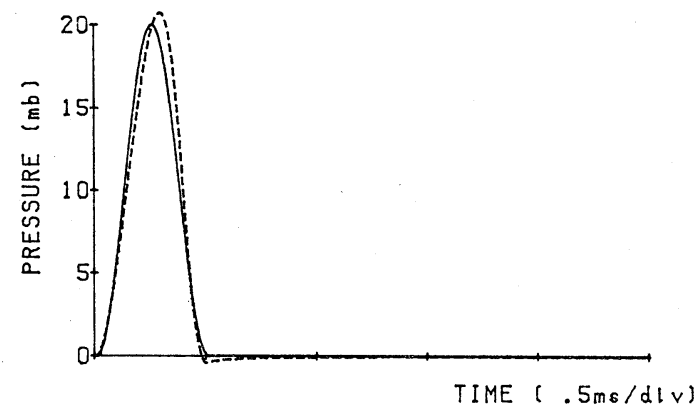

Fig. 2 Solid line is a squared since waveform and broken line is an inverse-propagated waveform to be generated at Mic. 1 position.

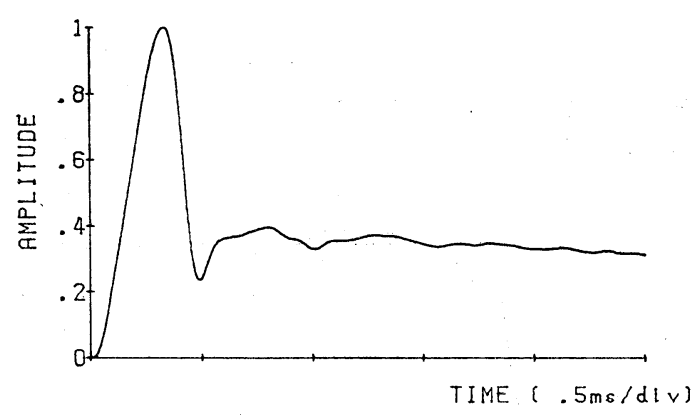

Fig. 3 Calculated waveform by Eq. (6) to generate a squared form of sinusoidal wave.

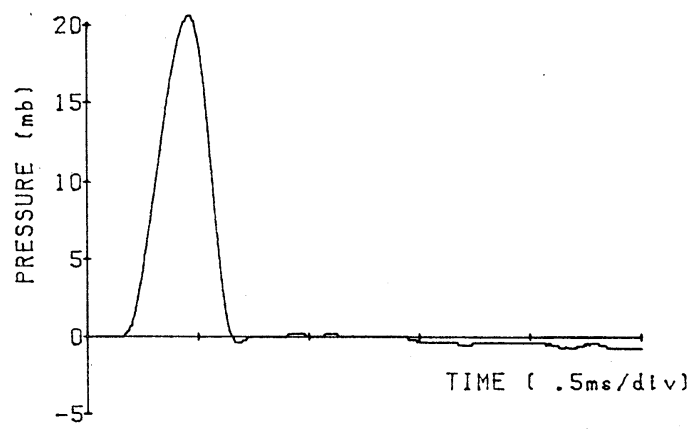

Fig. 4 Observed waveform by Mic. 1 for the input signal of Fig. 3.

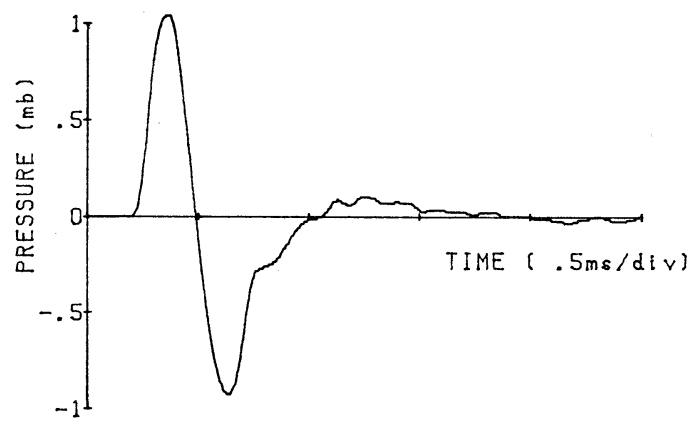

Fig. 5 Observed waveform by Mic. 2 for the input signal of Fig. 3.

\section{DISCUSSION}

\subsection{Simulation of Horn Characteristics}

It is obvious that the waveform shown in Fig. 5 is almost similar to a sinusoidal form, except the difference around the rear portion of the waveform. For this disagreement, it may be considered that the effect of the actual behaviour of sound in the horn, especially the reflection at the mouth of the horn, appears sensitively. Therefore, in order to estimate these effects quantitatively, the radiated waveform in free space was calculated from the input waveform at the throat of the horn by the following simulation, and compared with the experimental results in the previous chapter.

The simulation is based on a few assumptions for simplicity, which are the plane wave propagation through the horn and the linear reflection and transmission at boundaries. At first, the squared form of sinusoidal wave shown by the solid line in Fig. 2 is input to the position of the throat of the 


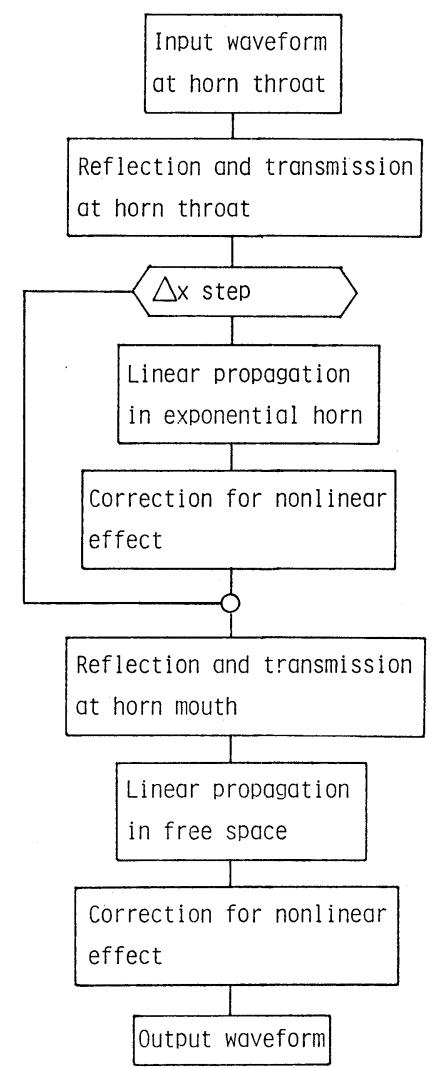

Fig. 6 Flow diagram for simulation of radiation of finite amplitude wave from the horn into free space.

horn and the transmitted waveform into the horn is calculated by the use of the reflection coefficient $R_{i}$ at the horn throat written as

$$
R_{i}=\frac{Z_{+}-\rho c}{Z_{+}+\rho c},
$$

where $Z_{+}$represents the acoustic impedances) concerning with the propagation of the wave in the horn to the positive direction which is the same direction as the incident wave. Next, the propagation in the horn is simulated by dividing the horn length into short intervals. The waveform variation is calculated by the propagation constant $\mu(\omega)$ obtained from the linear theory of the exponential horn written as

$$
\mu(\omega)=-\frac{m}{2}-\frac{j}{2} \sqrt{4 k^{2}-m^{2}},
$$

where $k=\omega / c$, and $m$ represents the flare constant of the horn. The effect of the nonlinear propagation of each short interval is corrected by the same way as the propagation through the pipe. Next, in order to obtain the waveform at the mouth of the horn, the reflection at the mouth is calculated by the use of the reflection coefficient $R_{o}$ for the particle velocity written as

$$
R_{o}=\frac{Z_{+}-Z_{r}}{Z_{r}+Z_{-}}
$$

where $Z_{r}$ and $Z_{-}$represent the radiation impedance of the virtual piston at the mouth and the acoustic impedance of the wave which propagates to the negative direction in the horn, respectively. At last, the radiated waveform $p(r, t)$ on axis in free field is calculated from the waveform $u(t)$ of the particle velocity at the mouth by the following equation,

$$
p(r, t)=\rho c\left\{u\left(t-\frac{r}{c}\right)-u\left(t-\frac{\sqrt{r^{2}+a^{2}}}{c}\right)\right\},
$$

where $r$ is the distance between the mouth and the observation point of Mic. 2 and $a$ is the radius of the mouth of the horn. And then the effect of the waveform distortion due to the nonlinear propagation is taken into account under the assumption that the waveform $p(r, t)$ linearly obtained by Eq. (10) propagates again like as a plane wave, but nonlinearly this time, from the mouth to the observation point. ${ }^{9)}$

The procedures of the simulation described above is shown in Fig. 6 and the result obtained is shown in Fig. 7. This figure gives the waveform at the point on axis apart $20 \mathrm{~cm}$ from the mouth. This simulated waveform agrees well with the experimental result shown in Fig. 5, and so it can be explained well that the disturbance around the rear portion of the waveform in Fig. 5 is attributed to

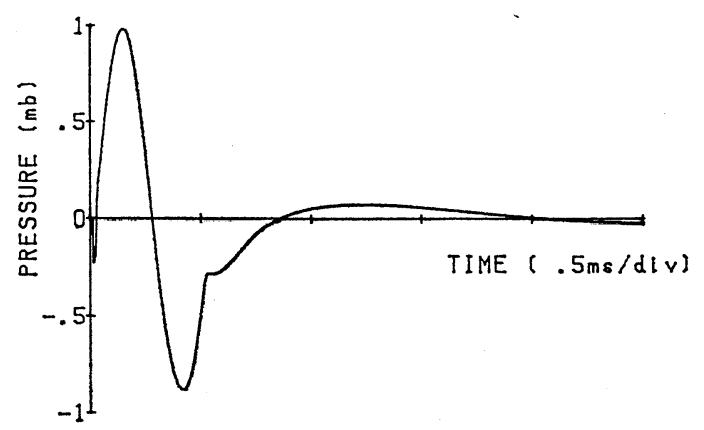

Fig. 7 Simulated waveform by taking account of the horn characteristics. 


\section{T. NAKAMURA and A. NAKAMURA: GENERATION OF SINUSOIDAL PULSE IN FREE SPACE}

the effect of the reflection at the mouth of the horn used in this experiment.

\subsection{Generation of Single Sinusoidal Pulse in Free Space}

As described in the previous section, it was confirmed that the method of the simulation was useful for the estimation of the waveform radiated from the exponential horn in spite of the rough approximations. And then it is expected to obtain the radiated waveform of a sinusoidal form with high accuracy by taking account of the horn characteristics. It means that the necessary waveform in the pipe is calculated from the waveform on axis in free space by performing the backward procedure of the simulation shown in Fig. 6. Results obtained are shown as follows. First, we plan to generate the sinusoidal waveform at the Mic. 2 position with the peak pressure of 1 mbar shown in Fig. 8, and therefore the waveform shown in Fig. 9 must be generated at the mouth of the horn. This is almost

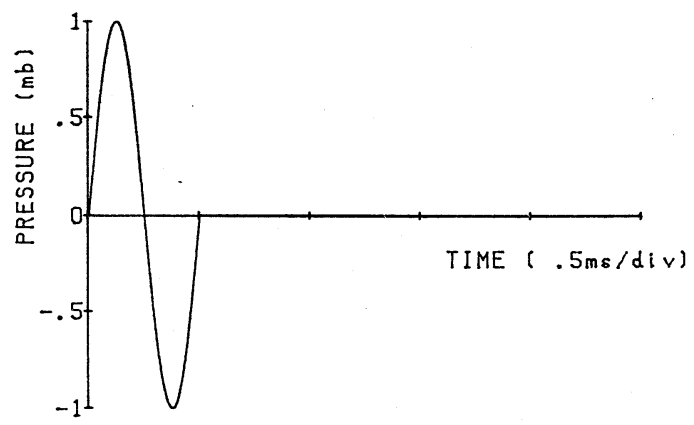

Fig. 8 A single sinusoidal waveform to be generated at Mic. 2 position.

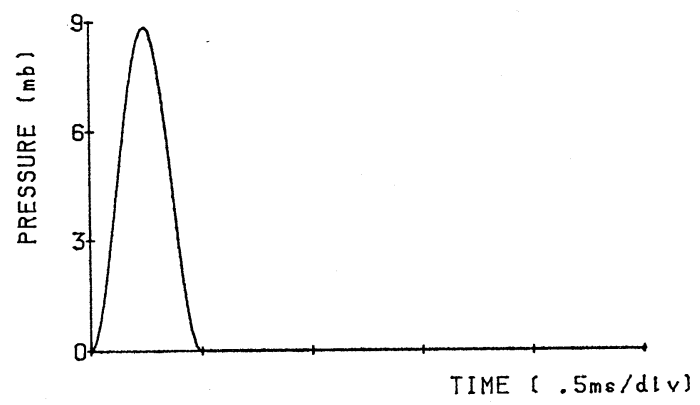

Fig. 9 A waveform simulated at the mouth of the horn. equivalent to the waveform of the solid line in Fig. 2. In order to realize the waveform shown in Fig. 9 at the mouth of the horn, the waveform given at the throat of the horn must be decided so as to eliminate the variation due to the characteristics of the exponential horn from the waveform of Fig. 9. The result is shown by the solid line in Fig. 10. Next, the waveform of the broken line in Fig. 10 is obtained by calculating the waveform distortion by the effect of nonlinear propagation to the inverse direction through the pipe between the Mic. 1 position and the throat of the horn. At last, the waveform shown in Fig. 11 is obtained by correcting the characteristic of the driver unit. When this waveform was input to the driver unit, the waveform shown in Fig. 12 was observed by Mic. 1 placed at the distance of $20 \mathrm{~cm}$ from the source and the waveform in Fig. 13 was observed by Mic. 2 placed at the point on axis apart $20 \mathrm{~cm}$ from the mouth of the horn. Then, the control of the waveform generation is performed at the Mic. 1 position

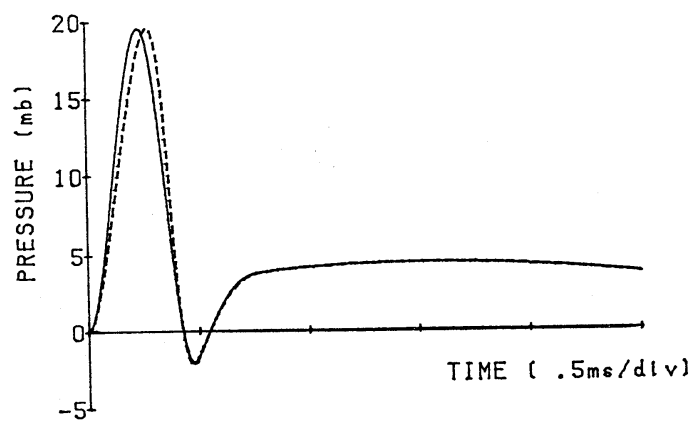

Fig. 10 Solid line is a waveform to be simulated at the throat of the horn and broken line is a waveform simulated at the Mic. 1 position.

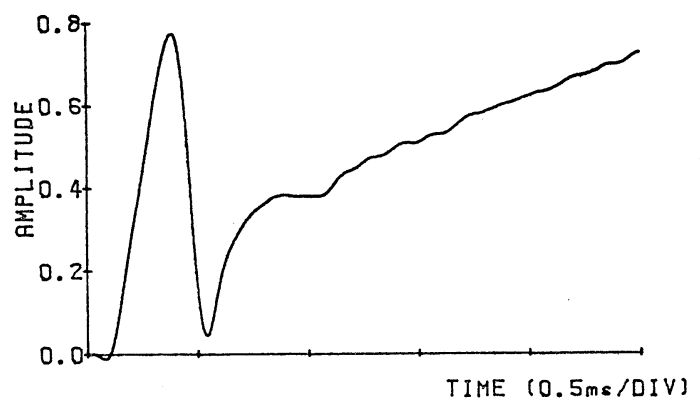

Fig. 11 Simulated waveform to generate a sinusoidal pulse in free space. 


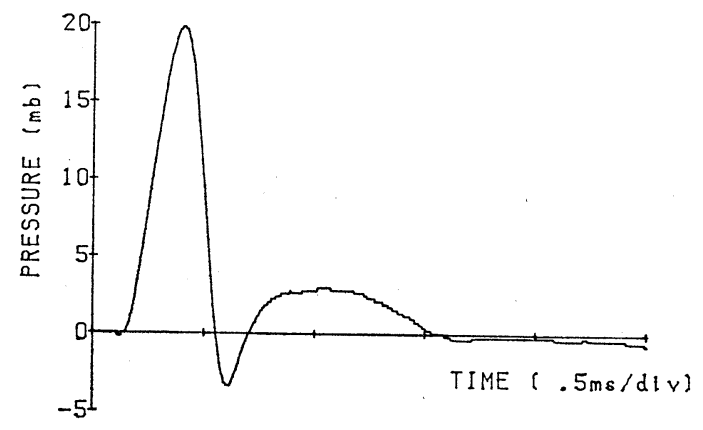

Fig. 12 Observed waveform by Mic. 1 for the input signal of Fig. 11.

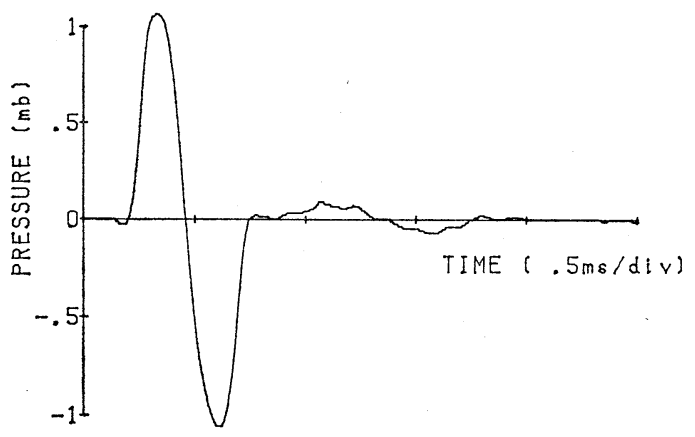

Fig. 13 Observed waveform by Mic. 2 for the input signal of Fig. 11.

so that the waveform of the broken line shown in Fig. 10 is obtained at this location. The waveform of Fig. 13 is good agreement with the sinusoidal form in Fig. 8 except a small disturbance following the first sinusoidal form. A clarified explanation of this disturbance has not yet been made. However, it may be considered that the neglection of the multiple reflection in the horn on the procedure of the simulation concerns with this disturbance.

\section{CONCLUSION}

In order to control the radiated waveform of the finite amplitude in free space, the waveform control in a pipe was performed by the use of the loudspeaker driven by the computed waveform and generated wave was radiated from an exponential horn connected to the end of the pipe. If the horn used in this experiment is assumed to be an ideal device for the impedance matching between the pipe and the free field, the integrated form of the wave appearing on axis in free space must be generated in the pipe, and then the squared form of sinusoidal wave was generated in the pipe in order to obtain the sinusoidal pulse in free space. However, the observed waveform in free space was different slightly from a sinusoidal form. As the results of the simulation for the sound transmission through the exponential horn, it became clear that the reflection of the mouth of the horn affected the radiated waveform in free space. In addition to this fact, it was found that horn characteristics shown by the incidence of the finite amplitude wave could be simulated by this method. Therefore, the waveform control in the pipe was performed by taking account of the effect of the horn and then the result obtained agreed approximately with the sinusoidal waveform expected.

From the results described above, we can indicate the possibility that the finite amplitude pulse with an arbitrary waveform, saying it differently, an arbitrary spectrum distribution can be generated in free space and therefore it is considered that this method may be used widely in the field of the general acoustic measurement as well as the nonlinear problems.

This work was partly supported by a Grant-inAid for Scientific Research from the Ministry of Education, Science and Culture of Japan.

\section{REFERENCES}

1) T. Nakamura and A. Nakamura, "New method for generation of finite amplitude sound pulse by the use of loudspeaker," J. Acoust. Soc. Jpn.(E) 3, 47-49 (1982).

2) T. G. Winter, J. Pereira, and J. B. Bender, "On driving a transducer to produce pulses shorter than the natural period of the transducer," Ultrasonics 13, 110-112 (1975).

3) R. Singh and T. Katra, "On the digital generation of an acoustic excitation impulse," J. Sound Vib. 58, 459-462 (1978).

4) A. Niedzwiecki and H. S. Ribner, "Subjective loudness of N-wave sonic booms," J. Acoust. Soc. Am. 64, 1617-1621 (1978).

5) J. C. Davis, J. McIntosh, and K. A. Mulholland, "The generation of short duration acoustic signals," J. Sound Vib. 76, 77-82 (1981).

6) I. Nakayama and A. Nakamura, "A theoreticalexperimental comparison of the transient waveform from circular plate excited by a triangular sound pulse," J. Sound Vib. 78, 446-451 (1981).

7) T. Nakamura, A. Nakamura, and R. Takeuchi, "Simulation for nonlinear propagation of finite 
T. NAKAMURA and A. NAKAMURA: GENERATION OF SINUSOIDAL PULSE IN FREE SPACE

amplitude sound wave through a circular pipe," Acustica 38, 331-333 (1977).

8) S. Yoshikawa, M. Murakami, and K. Ikegaya, "Consideration of exponential horn," J. Electr. Comm. Jpn. 45, 172-177 (1962) (in Japanese).
9) W. N. Cobb, "Diffraction of a plane $N$ wave of finite amplitude by a slit," Appl. Res. Lab. Univ. of Texas at Austin, Tech. Rep. ARL-TR-77-43 (1977). 